\title{
Extracorporeal membrane oxygenation for COVID-19: lessons learned
}

\author{
Gary S. Schwartz ${ }^{1}$, Britton Blough ${ }^{2}$, Kara Monday ${ }^{2}$, Rebecca Weddle ${ }^{2}$, Christopher Hebert ${ }^{3}$, \\ Cedric Spak ${ }^{4}$, Uriel Sandkovsky ${ }^{5}$, Robert L. Gottlieb ${ }^{6}$, Omar Hernandez ${ }^{7}$, Kaitlyn Lingle ${ }^{7}$, Dan Meyer ${ }^{8}$ \\ ${ }^{1}$ Department of Thoracic Surgery and Lung Transplantation, Baylor Scott and White Health, Dallas, TX, USA; ${ }^{2}$ North Texas Critical Care/Urgent \\ Surgical Associates, Dallas, TX, USA; ${ }^{3}$ Kidney and Hypertension Associates of Dallas, Dallas, TX, USA; ${ }^{4}$ Texas Centers for Infectious Disease \\ Associates, Dallas, TX, USA; ${ }^{5}$ North Texas Infectious Disease Consultants, Dallas, TX, USA; ${ }^{6}$ Department of Cardiology, Baylor Scott and White \\ Health, Dallas, TX, USA; ${ }^{7}$ Perfusion Services, Baylor Scott and White Health, Dallas, TX, USA; ${ }^{8}$ Department of Cardiac Surgery, Baylor Scott and \\ White Health, Dallas, TX, USA \\ Contributions: (I) Conception and design: All authors; (II) Administrative support: O Hernandez, K Lingle; (III) Provision of study materials or \\ patients: All authors; (IV) Collection and assembly of data: O Hernandez, K Lingle; (IV) Data analysis and interpretation: GS Schwartz, K Monday, \\ B Blough; (VI) Manuscript writing: All authors; (VII) Final approval of manuscript: All authors. \\ Correspondence to: Gary Schwartz, MD. Department of Thoracic Surgery and Lung Transplantation, Baylor University Medical Center, 3410 Worth \\ St Suite 545, Dallas, TX 75246, USA. Email: Gary.Schwartz@BSWHealth.org.
}

Background: The use of extracorporeal membrane oxygenation (ECMO) for the management of severe acute respiratory distress syndrome (ARDS) secondary to the 2019 coronavirus (COVID-19) has been described. However, the management of this complex patient population has evolved over the course of the pandemic, likely contributing to variable outcomes. We report our adaptive experience managing patients on venovenous (VV) ECMO from a single institution with a focus on the lessons learned.

Methods: From April through November 2020, 50 patients with COVID-19 ARDS were supported on VV ECMO. Patient characteristics were collected in an institutional database linked to the Extracorporeal Life Support Organization (ELSO) registry and included patient demographics, initial medical management, ECMO support details, ICU and hospital course, and short-term outcomes.

Results: As of December 1, 2020, 7 patients remained on ECMO support. Of the 43 patients who completed an ECMO run, 11 patients expired (25.6\%) and 32 patients (74.4\%) were weaned from ECMO. Thirty patients $(69.8 \%)$ were discharged alive from the hospital while 2 remained in the hospital and have been successfully weaned from mechanical ventilation.

Conclusions: Valuable lessons have been learned from our experience related to patient selection, cannulation strategies, anticoagulation, airway management, renal replacement therapy, physical therapy, pharmacological management, and resource utilization. Pre-pandemic VV ECMO guidelines and protocols have been dramatically modified based on these lessons, contributing to excellent survival. Management of this patient population is atypical and requires a constantly evolving approach.

Keywords: Extracorporeal membrane oxygenation (ECMO); coronavirus (COVID); acute respiratory distress syndrome (ARDS)

Received: 22 April 2021; Accepted: 07 September 2021; Published: 25 November 2021.

doi: $10.21037 /$ ccts-21-22

View this article at: https://dx.doi.org/10.21037/ccts-21-22 


\section{Introduction}

Severe cases of refractory acute respiratory distress syndrome (ARDS) secondary to the novel 2019 coronavirus (COVID-19) have been reported to be salvaged with extracorporeal membrane oxygenation (ECMO), most commonly venovenous (VV). Guidelines for application of this technology for this patient population has been well described (1-3), with survival to discharge approximately ranging from $45-63 \%(4,5)$. While national and international registries are continuously collecting data, crucial lessons have been learned regarding the management of this unique patient population and circulated in informal forums including organizational websites and webinars, social media, and word of mouth. However, the published literature has been limited due to the scarcity of data and overall small volume of experience, often pooling several centers' experiences (6-15).

Our institution was able to provide ECMO support on a large scale during this pandemic due to several factors. Fortunately, our region experienced a manageable volume and severity of patients and a delayed timeline relative to other national and international locations, allowing for lead-time preparation. A pre-existing infrastructure with significant ECMO capacity and experience was already in place, averaging 120 adult ECMO patients per year over the last 5 years and ELSO Center of Excellence Gold status. Finally, a refined transfer and transport system was already in place as a central hub for many regional centers.

We describe our initial experience with $50 \mathrm{VV}$ ECMO patients with COVID-19 ARDS at a single institution with a focus on the lessons learned in the management of this complex patient population with the goal of improving patient outcomes.

We present the following article in accordance with the STROBE reporting checklist (available at https://ccts. amegroups.com/article/view/10.21037/ccts-21-22/rc).

\section{Methods}

\section{Program development}

Initial discussion to implement a COVID-19-specific ECMO program began in February 2020 including bedding and staffing arrangements, equipment purchase and availability, and personnel training. Formal patient selection guidelines specific to COVID-19 were finalized in April 2020. Over the subsequent 8 months, a total of 50 patients with COVID-19 ARDS were cannulated for VV ECMO as a bridge to recovery.

Inclusion and exclusion criteria were consistent with ELSO guidelines, which included modifications or preexisting guidelines in expectation of a high volume of patient referrals and resource utilization (2). Specific modifications including a reduction in age from 70 to 60 years; time on mechanical ventilation $\leq 7$ days (previously 10 days); body mass index (BMI) less than $45 \mathrm{~kg} / \mathrm{m}^{2}$ (previously unrestricted); and the decision to not offer extracorporeal cardiopulmonary resuscitation (ECPR) unless cannulation was actively underway. Importantly, acute kidney injury (AKI) was not considered a contraindication, as a significant proportion of our pre-pandemic ECMO patients with simultaneous acute renal failure ultimately recovered.

\section{Clinical practice}

All patients were cared for in a dedicated wing of the Cardiothoracic Intensive Care Unit (ICU) with negative pressure capability and separated from the heart and lung transplant population. Initially, patients that could not safely be transported to our unit would not be considered candidates for ECMO support in order to limit potential staff exposure during cannulation and transport. However, as disease severity mandated cannulation in the Medical ICU for several patients, this policy was modified.

To reduce inter-operator variability and exposure risk, the decision was made to cannulate all patients in the same manner. All cannulators wore an N95 mask or a powered air purifying respirator (PAPR) with two layers of surgical gowns and gloves and shoe covers (Figure 1). Using ultrasound guidance, bifemoral VV cannulation was performed utilizing a 25-French or 23-French drainage cannula (Edwards Lifesciences Corp., Irvine, CA, USA) and a 21-French or 19-French Biomedicus long arterialstyle return cannula (Medtronic, Dublin, Ireland). Size decision was based on body surface area and cannulas were staggered by $5 \mathrm{~cm}$ to prevent recirculation. Based on early reports of COVID-19 myocarditis with rapid hemodynamic decompensation, initially a small gauge femoral arterial pressure line was simultaneously inserted for potential urgent conversion to venoarterial ECMO; fortunately, this was not encountered in our early experience and this practice was abandoned.

A combination of ECMO pumps and oxygenators were utilized based on patient volume and available disposable equipment. Pumps utilized included Rotaflow 


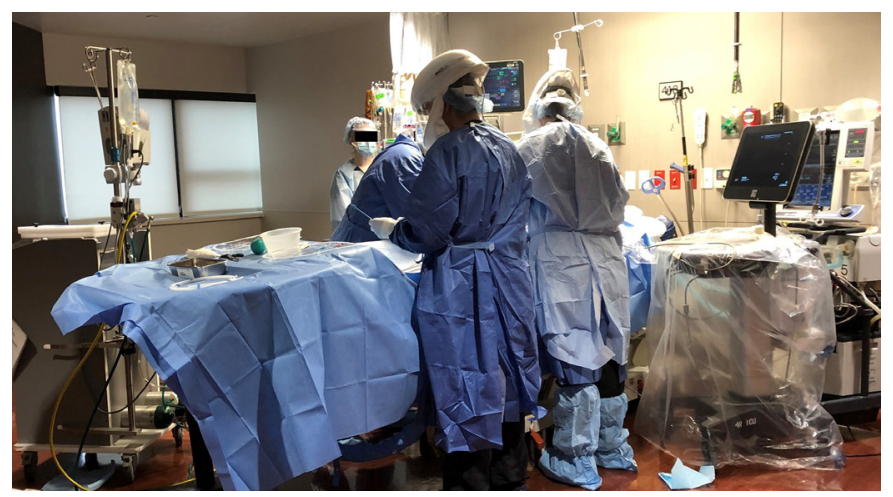

Figure 1 Cannulation utilizing full personal protective equipment. This image is published with the patient and all participants' consent.

(Getinge, Goteborg, Sweden), Cardiohelp (Getinge), and Centrimag (Abbott Laboratories, Sun Valley, CA, USA). Oxygenators included Quadrox (Getinge) and AMG (Abbott Laboratories). If continuous renal replacement therapy (CRRT) was necessary, a NxStage dialysis machine ( $\mathrm{NxStage}$ Medical Inc., Lawrence, MA, USA) was utilized with the filter in series with the ECMO circuit with venous return proximal to the oxygenator.

Patients with slow recovery with no evidence of improvement at 14 days and expected prolonged courses were revised to single-site right internal jugular dual lumen cannulation. This was reserved for patients that were satisfactorily awakened and rehabilitating, and it was felt that femoral cannulas were inhibiting forward progress. Patients that were rapidly recovering, slow to wake up, or able to ambulate with femoral cannulas were not revised. When necessary, this was performed electively in the operating room using fluoroscopy and transesophageal echocardiography and a Crescent ${ }^{\mathrm{TM}}$ dual-lumen cannula (Medtronic, Dublin, Ireland).

All patients were systemically anticoagulated with Heparin 5000 units intravenously at the time of cannulation. Pre-pandemic, our institution did not routinely anticoagulate all patients on VV ECMO; in fact, a randomized control trial is ongoing comparing subcutaneous heparin (5,000 units three times daily) to intravenous heparin titrated to an activated partial thromboplastin time (aPTT) of 40-60 seconds. However, as additional data regarding the thrombotic complications of COVID became apparent, the decision was made to exclude these patients from the ongoing trial and systemically anticoagulate. Indeed, once this was adopted and thrombotic issues were even more widely circulated in the medical literature, the goal of titration was arbitrarily increased to an aPTT of 55-65 seconds.

Patients that developed thrombocytopenia were tested for heparin-induced thrombocytopenia (HIT) antibodies, and if positive, switched to argatroban and further tested with serotonin release assay (SRA). Following decannulation, patients were transitioned to oral anticoagulation for 3 months.

Once cannulated, all patients would briefly remain paralyzed for bronchoscopy with bronchoalveolar lavage to evaluate for concomitant bacterial infection. The ventilator was disconnected to minimize aerosolization and potential staff exposure. Following bronchoscopy, the patient would be placed back on the ventilator and paralysis rapidly weaned to limit debility and initiate bedside physical therapy as early as possible.

Ventilator "rest settings" were initiated following cannulation. This consisted of a pressure control mode with a driving pressure of $10 \mathrm{cmH}_{2} \mathrm{O}$, respiratory rate of 10 breaths/minute, and a positive end-expiratory pressure (PEEP) of $10 \mathrm{cmH}_{2} \mathrm{O}$ for $\mathrm{BMI}<40 \mathrm{~kg} / \mathrm{m}^{2}$ and $15 \mathrm{cmH}_{2} \mathrm{O}$ for $\mathrm{BMI}>40 \mathrm{~kg} / \mathrm{m}^{2}$. Ventilator $\mathrm{FiO}_{2}$ would be weaned from $100 \%$ to a goal of $50 \%$ as tolerated.

Initially, tracheostomy would be performed only after a tracheal aspirate was negative for COVID-19 on two occasions and if still medically necessary. As the high need for tracheostomy became apparent, this practice was abandoned and all patients not extubatable within 48 hours of cannulation underwent early tracheostomy to facilitate sedation weaning and rehabilitation. Bedside passive physical therapy, tilt-table, and mobilization out of bed to chair and then ambulating with assistance was initiated as soon as medically feasible (Figure 2).

Pre-ECMO medical therapy varied widely. In general, therapies begun prior to ECMO initiation were 


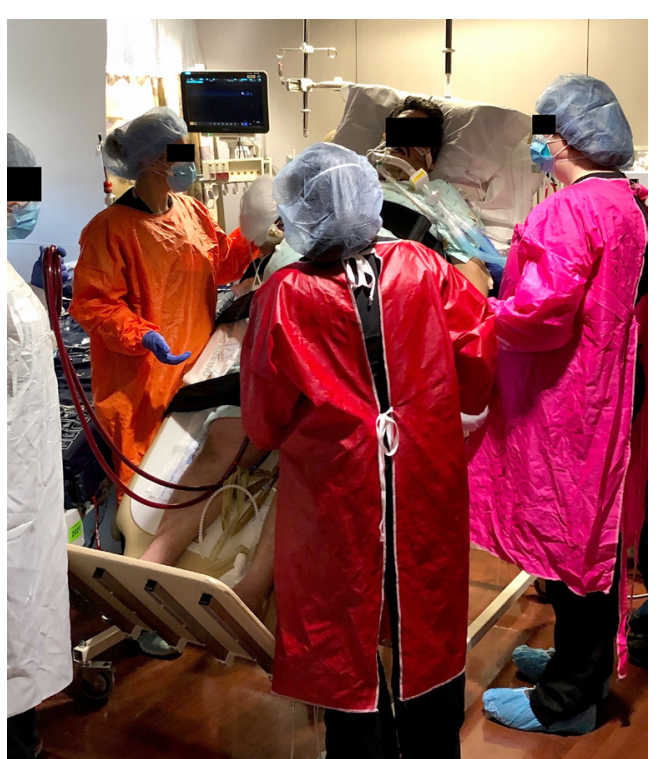

Figure 2 Tilt table mobilization of a 2019 coronavirus (COVID-19) acute respiratory distress syndrome (ARDS) patient on extracorporeal membrane oxygenation (ECMO). This image is published with the patient and all participants' consent.

continued. As additional data became available, systemic corticosteroids were administered to all patients. Antiviral and immunomodulatory therapies were continued on a clinical trial or as compassionate use. These included Remdesivir, Tocilizumab, Sirukumab, Leronlimab, and Gimsilumab. Additional doses of immunomodulatory therapy and high dose steroids were administered in select cases. Convalescent plasma was administered while on ECMO support.

\section{Data analysis}

Patient data were collected prospectively in our institutional database and reported to ELSO. This database was maintained in Microsoft Excel and reviewed retrospectively including statistical analyses of mean, median, and ranges. This was conducted in accordance with the Declaration of Helsinki (as revised in 2013) with approval of our Institutional Review Board (Protocol \#014-279); individual consent for this retrospective analysis was waived. All authors were responsible for designing the study and for compiling and analyzing the data. The manuscript was prepared by the first author and completed with input from all authors.

Demographic data included age, gender, race, BMI, and medical comorbidities. Disease-specific characteristics collected included time from diagnosis of COVID-19 to ECMO cannulation; time on mechanical support prior to ECMO cannulation; use of adjunctive ARDS therapies including paralysis, inhaled pulmonary vasodilators and prone positioning; pharmacological interventions including antiviral therapy, immunomodulatory therapy, systemic corticosteroids, convalescent plasma; and presence of additional organ failure. Inflammatory markers including d-dimer, C-reactive protein, ferritin, and interleukin-6 (IL6) were also systematically tracked.

Primary outcomes measures included rate of weaning from ECMO support and survival to discharge. Secondary outcome measures included time on ECMO support, ICU and hospital length of stay (LOS), and ECMO-related complications. These included AKI requiring CRRT; pneumothorax requiring tube thoracostomy; hemorrhagic complications including intracranial, gastrointestinal, cannulation site, and retroperitoneal; thrombotic including Heparin-induced thrombocytopenia (antibody positive) and circuit/cannula thrombosis requiring intervention; and infectious including secondary bacterial or co-viral pulmonary infection, bloodstream infection, cannulation site, and urinary tract infections.

\section{Results}

Demographic data is listed in Table 1. Median age was 50 years (range, $22-66$ years) with a male predilection (72\%) as reported worldwide and a Hispanic predominance (52\%) as reported nationally. Past medical history was significant for hypertension, diabetes, and morbid obesity in most patients. Three patients had pre-existing chronic kidney disease, with one end-stage on hemodialysis. Two patients were immunocompromised, one with HIV and one heart transplant recipient. Additional pre-ECMO organ failure was predominantly AKI necessitating CRRT which was present in $24 \%$ of patients. Median time on mechanical ventilation prior to ECMO initiation was 6 days, although this ranged from 0 to 20 days. Paralysis was utilized in $92 \%$ of patients and prone positioning in $72 \%(18 / 25)$. Inhaled pulmonary vasodilator therapy (prostaglandin or inhaled nitric oxide) was utilized in $12 \%(3 / 25)$.

Medical therapy was variable as was typically started prior to ECMO initiation. This included Hydroxychloroquine (24\%), Azithromycin (36\%), Remdesivir (64\%), immunomodulatory therapy (56\%), systemic corticosteroids (66\%), convalescent plasma (24\%), IVIG (4\%), and high 
Table 1 Demographics

\begin{tabular}{lc}
\hline Characteristics & Outcome \\
\hline Median age (IQR; range), years & $50(41-61 ; 22-67)$ \\
Male gender, $\mathrm{n}$ [\%] & $36[72]$ \\
Ethnicity, $\mathrm{n}$ [\%] & $26[52]$ \\
Hispanic & $12[24]$ \\
African American & $8[16]$ \\
White & $4[8]$ \\
Asian & $34.1(32.6-38.5)$ \\
Body-mass index, median (IQR), kg/m² & \\
Comorbidities incidence, $\mathrm{n}$ [\%] & $26[52]$ \\
Hypertension & $30[60]$ \\
Diabetes & $3[6]$ \\
Chronic kidney disease & $2[4]$ \\
Immunocompromised & $5[10]$ \\
Pre-ECMO hospital course & \\
Time (days) from admission to intubation & $2.4(1-6 ; 0-13)$ \\
(IQR; range) & \\
Time (days) from intubation to ECMO & \\
cannulation (IQR; range) & \\
Pre-ECMO interventions, $\mathrm{n}[\%]$ & $0-20)$ \\
Paralysis & \\
Prone positioning & \\
Renal replacement therapy & \\
\hline
\end{tabular}

dose Vitamin C (44\%). There was also significant variability in treatment over the study time period as additional data became available.

Primary outcome measures are demonstrated in Figure 3. Out of 50 patients cannulated during the study period, at the time of data analysis 7 patients remained on ECMO. Of the 43 patients who have completed an ECMO run, 11 patients expired (25.6\%) and 32 patients were successfully weaned $(74.5 \%)$. Of the 32 patients weaned from ECMO, 30 were discharged alive $(69.8 \%)$ and 2 remain in the hospital weaned from mechanical ventilation and awaiting discharge disposition. Of the 30 discharged alive, 12 were discharged home, 7 were discharged to acute rehabilitation, 8 were discharged to long-term acute care facilities, and 3 were transferred back to their referring hospitals.

Secondary outcome measures are listed in Table 2. Median time of ECMO support was 14 days (IQR 15.5). Median ICU and hospital LOS were 25.5 days (IQR 8.75) and 32 days (IQR 19.5), respectively. Sixteen patients (32\%) with prolonged courses were revised to a right internal jugular dual lumen cannula. Forty-nine patients (98\%) underwent tracheostomy. Three patients who were weaned from ECMO required recannulation several days later for profound hypercarbic respiratory acidosis; two were ultimately re-weaned from support and discharged alive and one remained in the hospital on extracorporeal support at the time of data analysis.

ECMO-related complications are also listed in Table 2. Of the 32 patients who developed AKI requiring RRT, 14 recovered renal function and came off dialysis prior to discharge. The remaining patients have either improved, requiring intermittent hemodialysis, or remain on ECMO

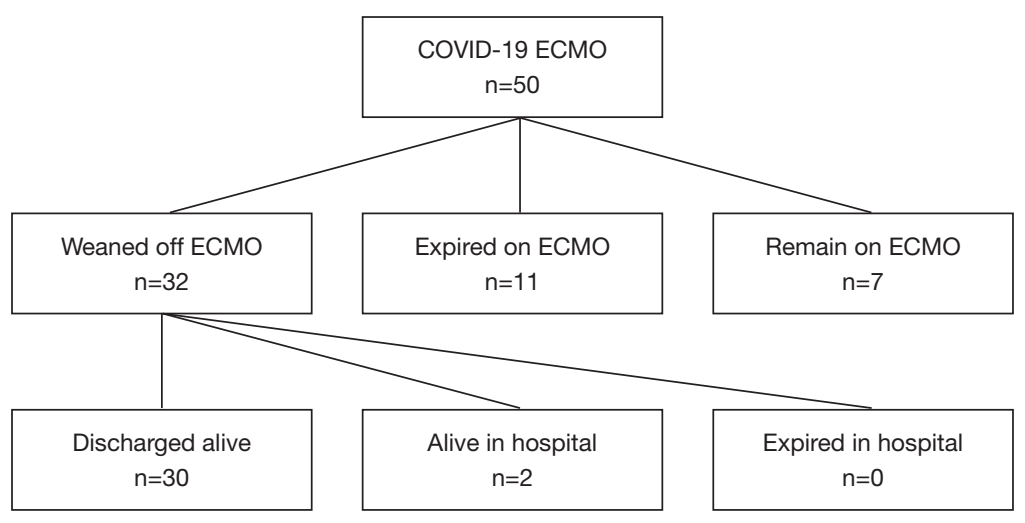

Figure 3 Primary outcomes. 
Table 2 Secondary outcomes

\begin{tabular}{lc}
\hline Characteristics & Outcome \\
\hline Median time of ECMO support (IQR), days & $14(15.5)$ \\
Median ICU LOS (IQR), days & $25.5(8.75)$ \\
Median hospital LOS (IQR), days & $32(19.5)$ \\
Complications, $n$ [\%] & \\
AKI & $32[64]$ \\
Pneumothorax & $11[22]$ \\
Hemorrhagic & \\
Intracranial & $6[12]$ \\
Gastrointestinal & $2[8]$ \\
Cannulation site & 0 \\
Retroperitoneal & $2[4]$ \\
Thrombotic & $2[4]$ \\
Heparin-induced thrombocytopenia & $6[12]$ \\
(anti-PF4) & $12[24]$ \\
Circuit/cannulation thrombosis & \\
DVT and/or PE & $2[4]$ \\
Infectious & $6[2]$ \\
Bacterial pneumonia & \\
Concomitant viral pneumonia & \\
Bloodstream & $12]$ \\
\hline
\end{tabular}

ECMO, extracorporeal membrane oxygenation; ICU, intensive care unit; LOS, length of stay; AKI, acute kidney injury; DVT, deep venous thrombosis; PE, pulmonary embolism.

with ongoing CRRT. Bleeding complications occurred in 9 patients. These included intracranial bleeding (6), gastrointestinal bleeding (2), and spontaneous retroperitoneal hematoma (2). Heparin induced thrombocytopenia (HIT) concerns arose in 12 patients, with a drop in platelets and positive HIT antibody testing prompting a switch to argatroban; ultimately, SRA testing was negative in all patients and they were either continued on argatroban or switched back to heparin at the physicians' discretion. Deep venous thrombosis (DVT) and pulmonary embolism (PE) despite systemic anticoagulation occurred in 6 and 2 patients, respectively. Cannula and/and circuit thrombosis requiring intervention occurred in 2 patients.
There was 1 surgical site infection. Bloodstream infections occurred in 6 patients.

\section{Discussion}

Valuable lessons were learned at our institution during the first wave of the pandemic as summarized in Table 3. Protocols and guidelines were prophylactically modified in preparation for a surge of patients requiring extracorporeal support and were continuously developed in real-time as institutional experience grew.

As in many therapeutic interventions, the importance of patient selection cannot be overemphasized. We arbitrarily decreased the inclusion age in our criteria in order to limit resource utilization; as can be seen from Table 1, several patients exceeded this age. As our experience grew but our case volume plateaued, we felt pre-COVID criteria were justified, as opposed to limiting this therapy based on theoretical resource limitations. Based on our series, advanced age did not impact mortality, as only one of the three deaths occurred in patients over the age of 60 . Although three patients had pre-existing renal disease, only one was on hemodialysis and he did not survive; this was subsequently adopted as an exclusion criterion.

Pre-pandemic, the ability to accept a patient for ECMO was the decision of the on-call cardiothoracic surgeon with a second opinion required for all denials and encouraged for the acceptance of high-risk patients. As the complexity of this patient population became more apparent, we instituted a requirement that all COVID-19 patients being considered for ECMO be discussed by a multidisciplinary team consisting of pulmonary medicine, surgical critical care, and cardiothoracic surgery. Additionally, a daily checkin between the COVID MICU and the COVID ECMO unit was instituted to identify tenuous patients and discuss the option of early ECMO initiation.

Given international reports of COVID-related myocarditis, the initial strategy had been to insert a femoral arterial line at the time of cannulation in case urgent conversion to venoarterial (VA) ECMO became necessary $(15,16)$. However, this did not occur in our series, and this practice was abandoned.

Airway interventions would initially be minimized to avoid aerosolization and health care worker infections, per societal guidelines (17). However, when necessary, full PPE including PAPR were utilized. Initially, tracheostomy would only be performed when a tracheal aspirate was negative; however, as the volume of patients grew as did 
Table 3 Lessons learned

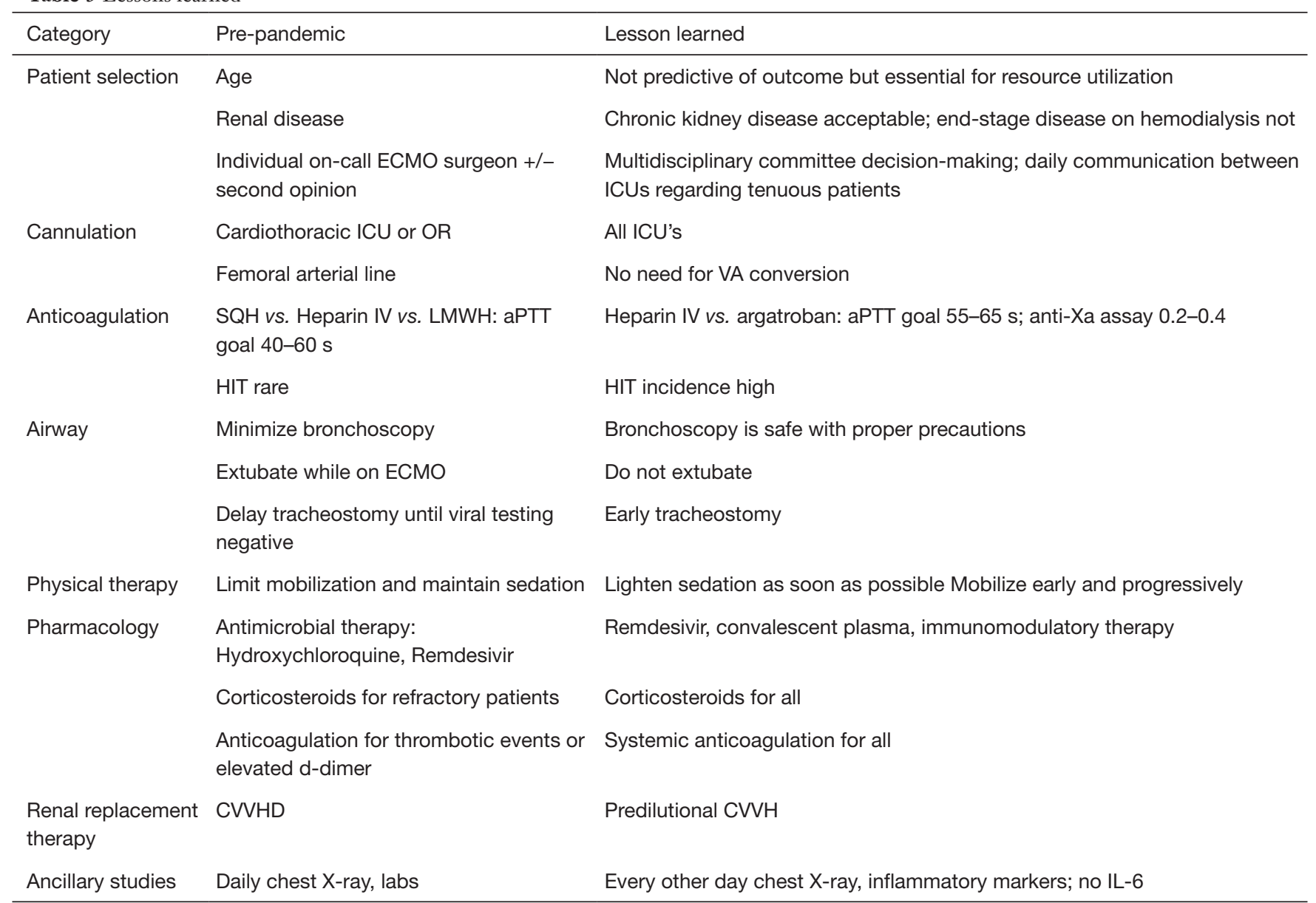

ECMO, extracorporeal membrane oxygenation; ICU, intensive care unit, OR, operating room; VA, venoarterial; SQH, subcutaneous Heparin; LMWH, low-molecular weight heparin; aPTT, activated partial thromboplastin time; HIT, heparin-induced thrombocytopenia; CVVHD, continuous veno-venous hemodialysis.

reports of safe tracheostomy techniques in actively infected patients (18-20), we began to perform tracheostomies independent of viral testing. Tracheostomy was performed in a strictly percutaneous technique, with a minimal skin incision and no subcutaneous dissection to avoid bleeding. Additionally, a purse-string suture was placed around the tracheostomy also to limit post-operative bleeding. Systemic anticoagulation was paused 4 hours before and after the procedure. In order to minimize aerosolization, patients were sedated and paralyzed and the ventilator stopped completely. Bedside percutaneous tracheostomy was performed utilizing bronchoscopic guidance with no change in technique as they were fully supported on extracorporeal support. Once the airway was secured, cuff inflated, and ventilator reconnected, mechanical ventilation was resumed.
Five patients were extubated while still on extracorporeal support. This was attempted in patients who had only recently been intubated with minimal time on mechanical ventilation, sedation, and paralysis. Unfortunately, this only proved successful in one patient, with the remaining four being reintubated and ultimately undergoing tracheostomy. Additionally, those that were extubated demonstrated significant air hunger, limiting improved mobility and contributed to abandoning this practice. Ultimately, the pendulum swung from attempting extubation, to avoiding extubation and proceeding with tracheostomy, to immediate early tracheostomy within 48 hours of cannulation.

Physical therapy evaluation and treatment was initiated as soon as possible. This began once an appropriate level of sedation weaning was accomplished, initially with bedside 
nursing performing passive therapy. Once more awake, gradual increased activity with physical therapists was performed, initially with tilt table, dangling at bedside, standing with assistance, and ultimately ambulating.

As mentioned, we elected to exclude COVID-19 patients from our ongoing institutional randomized trial comparing intravenous to subcutaneous anticoagulation for VV ECMO. In the non-ECMO COVID patient population at our institution, a d-dimer level of $3 \mathrm{mcg} / \mathrm{mL}$ was utilized to trigger anticoagulation, and this was surpassed in all patients requiring ECMO. A preexisting goal was an aPTT of 40-60 seconds; we elected to err on the higher side with an arbitrarily chosen goal of 55-65 seconds. Interestingly, 12 patients developed severe thrombocytopenia concerning for HIT and 10 of these developed anti-PF4 antibodies via enzyme-linked immunosorbent assay, yet none of them had positive serotonin release functional assay.

Bleeding complications were significant but as expected for ECMO patients. The rate of intracranial bleed appears is higher than the general ECMO population but lower than reported in other COVID ECMO series. Neither retroperitoneal bleed was cannulation related, and in fact one occurred five days after separation from extracorporeal support. One was managed with intra-arterial embolization and ultimately needed surgical evacuation, and the other managed with anticoagulation reversal alone.

The COVID-19 pandemic has forced innovation in the delivery and resource utilization of CRRT. In our series, $32 / 50$ patients undergoing extracorporeal life support developed AKI necessitating CRRT. The decision to start CRRT was typically due to volume overload with severe lung injury and hypoxemia or due to worsening acidosis in the setting of oliguria and profound respiratory acidosis. Despite excellent access flows with CRRT and systemic heparinization, we had several issues maintaining circuit patency on CRRT due to the thrombophilic nature of COVID-19. When our patients were changed from CVVHD to predilutional CVVH, we had significantly less clotting and an overall easier time maintaining circuit patency. There were no problems keeping adequate clearance on 24-hour CVVH.

Recognizing the significant resource utilization of these patients, a stream-lined order set was initiated to limited unnecessary testing. Chest radiographs and inflammatory markers including d-dimer, C-reactive protein, and ferritin are performed every other day. IL- 6 was tested at baseline and weekly thereafter given the limited clinical utility; ultimately this was abandoned altogether as it had little impact on patient care. Given the inability to visit in person, daily phone calls were made between the treatment team and family members for clinical updates, as well as video conferencing when able.

Adjunctive pharmacological therapies included antiviral therapies (within and independent of clinical trials), systemic corticosteroids, immunomodulatory therapy, and convalescent plasma. Selection of these therapies often occurred prior to referral for ECMO support and were executed in conjunction with the infectious disease service. Effect of these therapies on outcome remain to be determined by larger studies, with this cohort too small to comment on contribution to the overall results.

\section{Conclusions}

The utility of VV ECMO in patients with COVID-19 ARDS has proven very promising, with excellent outcomes to date. Standardization of patient selection, cannulation technique, and critical care management in the setting of an experienced center has made this possible. New challenges due to this disease process were encountered and managed in real time. The ultimate volume and duration of this pandemic remains to be seen, and the availability of ECMO support will be limited by the geographic and institutional resources available. However, the clinical utility is no doubt supportive of utilizing this therapy in ARDS secondary to COVID-19.

\section{Acknowledgments}

We are indebted to the nursing and ECMO specialist teams of the Cardiothoracic Surgery ICU at Baylor University Medical Center for the unparalleled level of care they provide to our patients daily.

Funding: None.

\section{Footnote}

Reporting Checklist: The authors have completed the STROBE reporting checklist. Available at https://ccts. amegroups.com/article/view/10.21037/ccts-21-22/rc

Data Sharing Statement: Available at https://ccts.amegroups. com/article/view/10.21037/ccts-21-22/dss

Peer Review File: Available at https://ccts.amegroups.com/ 
article/view/10.21037/ccts-21-22/prf

Conflicts of Interest: All authors have completed the ICMJE uniform disclosure form (available at https:// ccts.amegroups.com/article/view/10.21037/ccts-21-22/ coif). GSS serves as an unpaid editorial board member of Current Challenges in Thoracic Surgery from December 2019 to November 2021. GSS reports receiving honorarium from Abbott Technologies as a member of the speaking bureau and advisory medical board. RLG reported being a study investigator for Gilead Sciences, Eli Lilly, Kinevant (Roivant), Johnson and Johnson, Regeneron and Roche/ Genentech, receiving consulting fees from Gilead Sciences, and an advisor/review panel member for Eli Lilly, Gilead Sciences, GSK, Roche/Genentech, Roivant Sciences and Johnson and Johnson and reported receiving other financial or material support (Gift in kind to Baylor Scott and White Research Institute for NCT03383419) from Gilead Sciences. The other authors have no conflicts of interest to declare.

Ethical Statement: The authors are accountable for all aspects of the work in ensuring that questions related to the accuracy or integrity of any part of the work are appropriately investigated and resolved. This was conducted in accordance with the Declaration of Helsinki (as revised in 2013) with approval of our Institutional Review Board (Protocol \#014-279); individual consent for this retrospective analysis was waived.

Open Access Statement: This is an Open Access article distributed in accordance with the Creative Commons Attribution-NonCommercial-NoDerivs 4.0 International License (CC BY-NC-ND 4.0), which permits the noncommercial replication and distribution of the article with the strict proviso that no changes or edits are made and the original work is properly cited (including links to both the formal publication through the relevant DOI and the license). See: https://creativecommons.org/licenses/by-nc-nd/4.0/.

\section{References}

1. Bartlett RH, Ogino MT, Brodie D, et al. Initial ELSO Guidance Document: ECMO for COVID-19 Patients with Severe Cardiopulmonary Failure. ASAIO J 2020;66:472-4. Erratum in: ASAIO J 2020;66:e113.

2. Shekar K, Badulak J, Peek G, et al. Extracorporeal Life Support Organization Coronavirus Disease 2019 Interim
Guidelines: A Consensus Document from an International Group of Interdisciplinary Extracorporeal Membrane Oxygenation Providers. ASAIO J 2020;66:707-21.

3. Hoyler MM, Kumar S, Thalappillil R, et al. VV-ECMO usage in ARDS due to COVID-19: Clinical, practical and ethical considerations. J Clin Anesth 2020;65:109893.

4. Barbaro RP, MacLaren G, Boonstra PS, et al. Extracorporeal membrane oxygenation support in COVID-19: an international cohort study of the Extracorporeal Life Support Organization registry. Lancet 2020;396:1071-8.

5. Lorusso R, Combes A, Coco VL, et al. ECMO for COVID-19 patients in Europe and Israel. Intensive Care Med 2021;47:344-8.

6. Zeng Y, Cai Z, Xianyu Y, et al. Prognosis when using extracorporeal membrane oxygenation (ECMO) for critically ill COVID-19 patients in China: a retrospective case series. Crit Care 2020;24:148.

7. Hartman ME, Hernandez RA, Patel K, et al. COVID-19 Respiratory Failure: Targeting Inflammation on VVECMO Support. ASAIO J 2020;66:603-6.

8. Li X, Guo Z, Li B, et al. Extracorporeal Membrane Oxygenation for Coronavirus Disease 2019 in Shanghai, China. ASAIO J 2020;66:475-81.

9. Jacobs JP, Stammers AH, St Louis J, et al. Extracorporeal Membrane Oxygenation in the Treatment of Severe Pulmonary and Cardiac Compromise in Coronavirus Disease 2019: Experience with 32 Patients. ASAIO J 2020;66:722-30.

10. Sultan I, Habertheuer A, Usman AA, et al. The role of extracorporeal life support for patients with COVID-19: Preliminary results from a statewide experience. J Card Surg 2020;35:1410-3.

11. Taniguchi H, Ogawa F, Honzawa H, et al. Venovenous extracorporeal membrane oxygenation for severe pneumonia: COVID-19 case in Japan. Acute Med Surg 2020;7:e509.

12. Henry BM, Lippi G. Poor survival with extracorporeal membrane oxygenation in acute respiratory distress syndrome (ARDS) due to coronavirus disease 2019 (COVID-19): Pooled analysis of early reports. J Crit Care 2020;58:27-8.

13. Osho AA, Moonsamy P, Hibbert KA, et al. Veno-venous Extracorporeal Membrane Oxygenation for Respiratory Failure in COVID-19 Patients: Early Experience From a Major Academic Medical Center in North America. Ann Surg 2020;272:e75-8.

14. Yang X, Cai S, Luo Y, et al. Extracorporeal Membrane 
Oxygenation for Coronavirus Disease 2019-Induced Acute Respiratory Distress Syndrome: A Multicenter Descriptive Study. Crit Care Med 2020;48:1289-95.

15. Chow J, Alhussaini A, Calvillo-Argüelles O, et al. Cardiovascular Collapse in COVID-19 Infection: The Role of Venoarterial Extracorporeal Membrane Oxygenation (VA-ECMO). CJC Open 2020;2:273-7.

16. Bemtgen X, Krüger K, Supady A, et al. First Successful Treatment of Coronavirus Disease 2019 Induced Refractory Cardiogenic Plus Vasoplegic Shock by Combination of Percutaneous Ventricular Assist Device and Extracorporeal Membrane Oxygenation: A Case Report. ASAIO J 2020;66:607-9.

17. Wahidi MM, Shojaee S, Lamb CR, et al. The Use of

doi: $10.21037 /$ ccts-21-22

Cite this article as: Schwartz GS, Blough B, Monday K, Weddle R, Hebert C, Spak C, Sandkovsky U, Gottlieb RL, Hernandez O, Lingle K, Meyer D. Extracorporeal membrane oxygenation for COVID-19: lessons learned. Curr Chall Thorac Surg 2021;3:34.
Bronchoscopy During the Coronavirus Disease 2019 Pandemic: CHEST/AABIP Guideline and Expert Panel Report. Chest 2020;158:1268-81.

18. Angel L, Kon ZN, Chang SH, et al. Novel Percutaneous Tracheostomy for Critically Ill Patients With COVID-19. Ann Thorac Surg 2020;110:1006-11.

19. Prabhakaran K, Malcom R, Choi J, et al. Open tracheostomy for COVID-19-positive patients: A method to minimize aerosolization and reduce risk of exposure. J Trauma Acute Care Surg 2020;89:265-71.

20. McGrath BA, Brenner MJ, Warrillow SJ, et al. Tracheostomy in the COVID-19 era: global and multidisciplinary guidance. Lancet Respir Med 2020;8:717-25. 